\title{
Functional and Structural Brain Plasticity Enhanced by Motor and Cognitive Rehabilitation in Multiple Sclerosis
}

\author{
Luca Prosperini,, Maria Cristina Piattella, ${ }^{1}$ Costanza Giannì, ${ }^{2}$ and Patrizia Pantano ${ }^{1,3}$ \\ ${ }^{1}$ Department of Neurology and Psychiatry, Sapienza University, Viale dell'Università 30, 00185 Rome, Italy \\ ${ }^{2}$ Department of Radiological Sciences, Oncology and Pathology, Sapienza University, Rome, Italy \\ ${ }^{3}$ IRCCS Neuromed, Pozzilli, Italy \\ Correspondence should be addressed to Patrizia Pantano; patrizia.pantano@uniromal.it
}

Received 12 February 2015; Accepted 16 April 2015

Academic Editor: Michael G. Stewart

Copyright (C) 2015 Luca Prosperini et al. This is an open access article distributed under the Creative Commons Attribution License, which permits unrestricted use, distribution, and reproduction in any medium, provided the original work is properly cited.

\begin{abstract}
Rehabilitation is recognized to be important in ameliorating motor and cognitive functions, reducing disease burden, and improving quality of life in patients with multiple sclerosis (MS). In this systematic review, we summarize the existing evidences that motor and cognitive rehabilitation may enhance functional and structural brain plasticity in patients with MS, as assessed by means of the most advanced neuroimaging techniques, including diffusion tensor imaging and task-related and resting-state functional magnetic resonance imaging (MRI). In most cases, the rehabilitation program was based on computer-assisted/video game exercises performed in either an outpatient or home setting. Despite their heterogeneity, all the included studies describe changes in white matter microarchitecture, in task-related activation, and/or in functional connectivity following both task-oriented and selective training. When explored, relevant correlation between improved function and MRI-detected brain changes was often found, supporting the hypothesis that training-induced brain plasticity is specifically linked to the trained domain. Small sample sizes, lack of randomization and/or an active control group, as well as missed relationship between MRI-detected changes and clinical performance, are the major drawbacks of the selected studies. Knowledge gaps in this field of research are also discussed to provide a framework for future investigations.
\end{abstract}

\section{Introduction}

Multiple sclerosis (MS) is a long-lasting disease typically affecting young adults characterized by the presence of multifocal inflammatory demyelinated plaques distributed over time and space within the central nervous system (CNS) [1]. Pathological features of MS include breakdown of the blood-brain barrier, multifocal inflammation, demyelination, oligodendrocyte loss, reactive gliosis, and various degrees of axonal damage, ranging from transient dysfunction to irreversible loss, even at early stages of the disease [2]. Although acute inflammation usually causes reversible neurological dysfunction, MS relapses may also lead to residual irreversible disability involving both motor and cognitive functions $[3,4]$.

In the last decade, improved diagnostic criteria and availability of effective disease-modifying drugs have led to a paradigm shift towards earlier diagnosis and treatment $[5,6]$. In spite of this, either the actually available disease-modifying treatment or the other pharmacological approaches have little or no impact on permanent impairments, with motor symptoms and cognitive deficit reported, respectively, by $45-90 \%$ and $40-65 \%$ of patients throughout their disease, with a certain degree of overlap [7-9].

Consequently, the management of motor and cognitive disturbances still relies on rehabilitative strategies [10, 11], which have been reported to be effective in ameliorating these functional domains, suggesting that remediation/compensation may occur into even damaged brain structures [12]. This may imply that rehabilitation is able to enhance neuroplasticity, that is, the intrinsic property of the CNS to structurally and functionally adapt itself in response to external stimuli, environmental changes, or injuries [13]. While in healthy individuals the plasticity represents the basis 
of brain development, learning, and memory, in the context of MS this term encompasses molecular, synaptic, cellular events and even reorganization of the brain cortex or fibers that result in recovery of function after an acute or chronic damage [14].

The most promising advanced magnetic resonance imaging (MRI) techniques for investigating brain plasticity are the functional MRI (fMRI) and diffusion tensor imaging (DTI) [15]. The fMRI is based on the detection of changes in the blood oxygenation level-dependent (BOLD) signal, which is in turn affected by changes in neural activity in a specific brain region and the underlying physiology or pathology. Changes in BOLD signals can be investigated during the execution of a specific task (e.g., simple motor activity, sensory stimulation, and cognitive effort) [16] or at rest to explore the functional connectivity (FC), that is, the functional interaction between different brain regions [17]. The DTI is a method to assess myelin integrity in vivo, providing information on the integrity of the myelin-axon unit based on the directional asymmetry of water diffusion, that is, the so-called fractional anisotropy (FA) [18]. The FA value is determined by the ratio of axial diffusivity (AD) and radial diffusivity $(\mathrm{RD})$, and decreased $\mathrm{AD}$ and increased $\mathrm{RD}$ are considered as markers of axonal damage and demyelination, respectively, thus representing parameters that are sensitive to underlying pathological processes of MS [19].

Using these advanced MRI techniques, it has been recently demonstrated that rapid-onset plasticity and functionally relevant chronic reorganization processes are preserved even in the most advanced stage of the disease and that these phenomena are functionally relevant to maintain motor and cognitive function $[16,20,21]$.

All these findings support the hypothesis that neuroplasticity may be enhanced by rehabilitation [12]. In this view, advanced MRI may address knowledge gaps between the observed clinical improvement and the neural mechanisms underlying the improved function after rehabilitation, providing a powerful tool to investigate functional and structural brain changes related to recovery of function [22]. However, only few studies have investigated the mechanisms of rehabilitation-induced neuroplasticity so far, providing fragmented and incomplete data, in spite of the fact that rehabilitation is recognized as having a key role in the management of patients with MS [23].

Therefore, in this systematic review, we sought to summarize the existing MRI-based evidences that motor and cognitive rehabilitation may induce functional and structural plasticity into the brain of patients with MS.

\section{Methods}

Search Strategy and Article Selection. According to the Preferred Reporting Items for Systematic Review and MetaAnalyses (PRISMA) statement [24], two electronic databases (PubMed and Scopus) were searched for English-language articles focusing on human studies.

The search was run using the following terms: ("training" OR "rehabilitation") AND "imaging" AND "multiple sclerosis" [All Fields]. No article type limitations or time period restrictions were applied, and the latest search was undertaken on January 20, 2015.

Attempts to identify further articles were done by searching the references of the studies. We were not familiar with any study currently in progress that could be considered for inclusion, except for one study from our group recently submitted. Published conference abstracts, articles not available in English, and those including patients also affected by neurological conditions other than multiple sclerosis were excluded.

To fit the main purpose of this systematic review, that is, summarize the existing MRI-based evidences of rehabilitation-enhanced functional and structural plasticity in MS, we also excluded studies whose training was based on short-term learning, those in which MRI was used to predict the outcome of rehabilitation, and those in which the occurrence of brain plasticity was assessed by means of techniques other than MRI (e.g., transcranial magnetic stimulation, electroencephalogram, etc.).

Abstracts of resulting articles were then examined in order to select studies that met eligibility criteria. To assess eligibility, two investigators (Surnames are provided) independently searched for articles, and agreement between them was required in order to include an article. In case of disagreement, the decision was made by the most experienced author (P. Pantano) after reading the whole article.

First author, year of publication, sample size, study design, type and duration of intervention, clinical and MRI outcome measures, and interpretation of findings were extracted from included articles and recorded on an electronic spreadsheet (M. C. Piattella).

Methodological quality of included articles was assessed using the scale developed by the Physiotherapy Evidence Database (PEDro) initiative [25]. The purpose of the PEDro scale is to determine the external validity (criterion 1), internal validity (criteria 2-9), and statistical soundness (criteria 10-11) of a study included in a systematic review. Studies scoring equal or above 9 on the PEDro scale were considered methodologically "excellent," studies ranging from 6-8 were considered "good," studies scoring 4-5 were of "fair" quality, studies scoring below 4 were felt to be of "poor" quality.

\section{Results}

The strategy search initially yielded 216 and 231 articles in the PubMed and Scopus databases, respectively; additional 10 articles were found from other sources (references of selected papers). After applying the inclusion/exclusion criteria and checking full-text articles for eligibility, a total of 16 articles were included in the qualitative synthesis, as shown in the flow diagram in Figure 1.

Six studies investigated whether motor rehabilitation strategies enhance brain plasticity, as evaluated by either taskrelated fMRI $(n=2)[26,27]$, DTI $(n=3)$ [28-30], or both techniques $(n=1)$ [31] (see Table 1$)$.

Ten studies investigated whether cognitive rehabilitation strategies enhance brain plasticity, as evaluated by either taskrelated fMRI $(n=5)$ [32-35, 37] (see Table 2) or restingstate (RS)-fMRI $(n=4)$ (see Table 3) [38-41]; just one article 


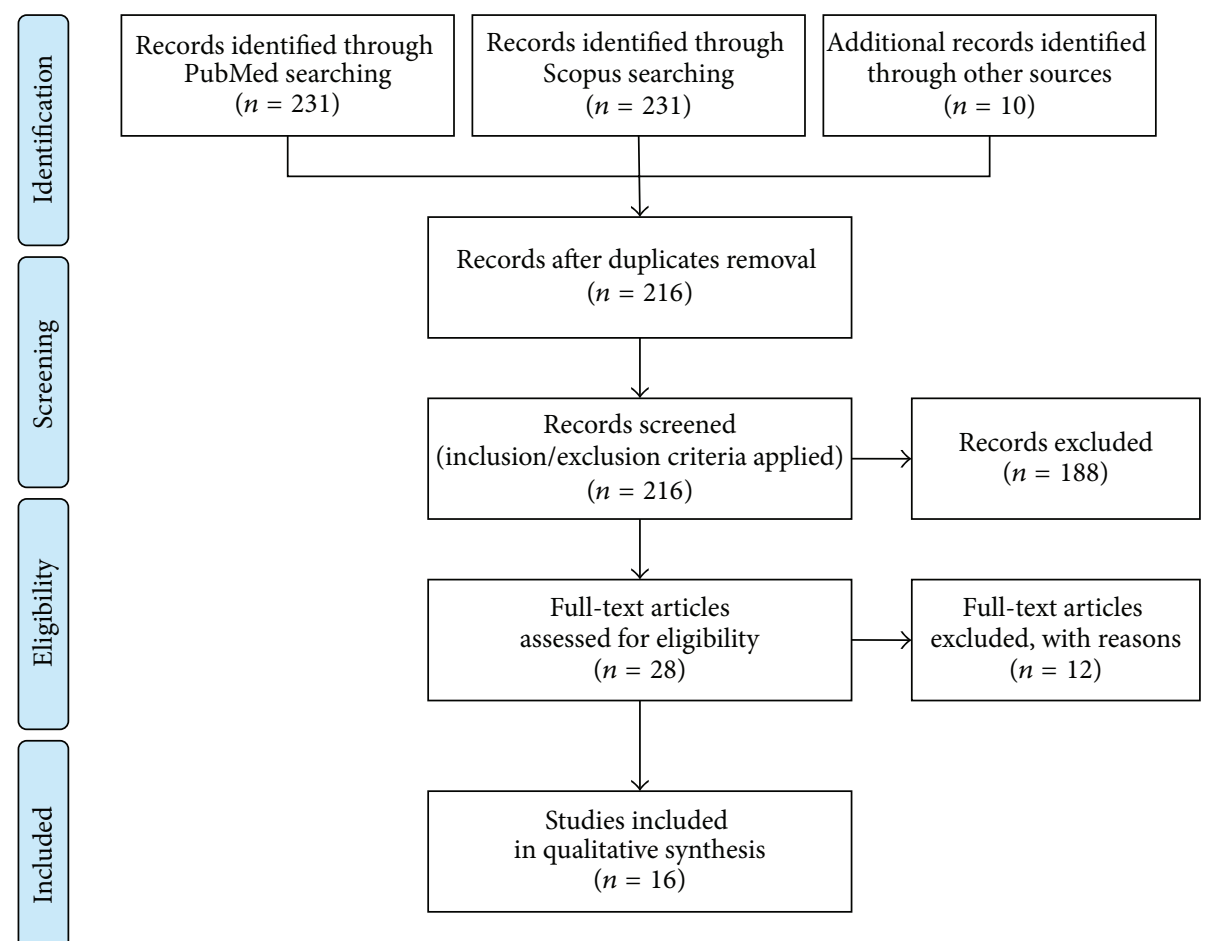

FIGURE 1: Flow diagram mapping the review according to PRISMA statement [24].

provided findings by combining task-related fMRI and RSfMRI with structural MRI for mapping changes in white matter (WM) and grey matter (GM) (included in Table 2) [36].

The qualitative assessment of included studies is shown in Table 4. Data from PEDro scale showed that selected article scored between 4 and 10 of 11 total points. Studies based on nonrandomized design [26-28, 31-33, 35, 40] or those without an alternative "sham" training as control group obtained lower scores [30, 36, 38, 41].

3.1. Brain Plasticity Enhanced by Motor Rehabilitation. The first attempt to demonstrate the occurrence of brain plasticity following motor rehabilitation was done by Rasova and colleagues [26] who selected (without randomization) 28 patients with MS. Of them, 17 received a 2-month outpatient physiotherapy program (1-hour sessions, twice per week) based on sensorimotor learning and adaptation by combining different disciplines (Vojta's reflective locomotion, Bobath concept, sensorimotor stimulation, proprioceptive neuromuscular facilitation, Burger concept, and yoga), while the remaining 11 patients did not undergo any special training. A control group of 13 healthy subjects was also enrolled to investigate whether rehabilitation might lead brain function to approach "standard" (i.e., values found in healthy group), as evaluated by task-related fMRI consisting of repetitive thumb and index flexions at a 3-second frequency, according to a visual stimulus. Each group was scanned twice at enrolment and 2 months later. Although a relevant clinical improvement was found in the active group when compared with the control group, the authors failed to demonstrate between-group differences in the amplitude of fMRI signal of four areas contributing to sensorimotor learning (primary sensorimotor cortex, supplementary motor cortex, nucleus dentatus, and putamen), as well as increased interhemispheric dependence. Moreover, there was no relationship between changes in clinical parameters and in brain activation. The authors concluded that the unpredictable course of the disease and the heterogeneous, symptom-tailored rehabilitation strategy hampered the detection of changes at group-level in fMRI activation, questioning about the appropriateness of fMRI for investigating motor plasticity.

The same group explored the impact of operator-assisted facilitation physiotherapy on microstructural properties of the corpus callosum in 11 right-handed patients with MS [28]. They were scanned in two separate occasions 1 month apart (run-in period) and then after the 2-month rehabilitation (2-hours per week). Increased callosal FA, reduced mean diffusivity $(\mathrm{MD})$ and $\mathrm{RD}$ were found after the intervention (by approaching the values of 11 healthy controls), while no difference was observed during the run-in period. Improved scores at the Paced Auditory Serial Addition Test (PASAT) and a trend towards an improvement of the Expanded Disability Status Scale (EDSS) score are reported by the authors, but the relationship between clinical improvement and MRI changes was not reported.

More recently, a similar study was conducted to investigate the immediate and long-term effects of a 2-month motor program activation therapy (1-hour sessions, twice 


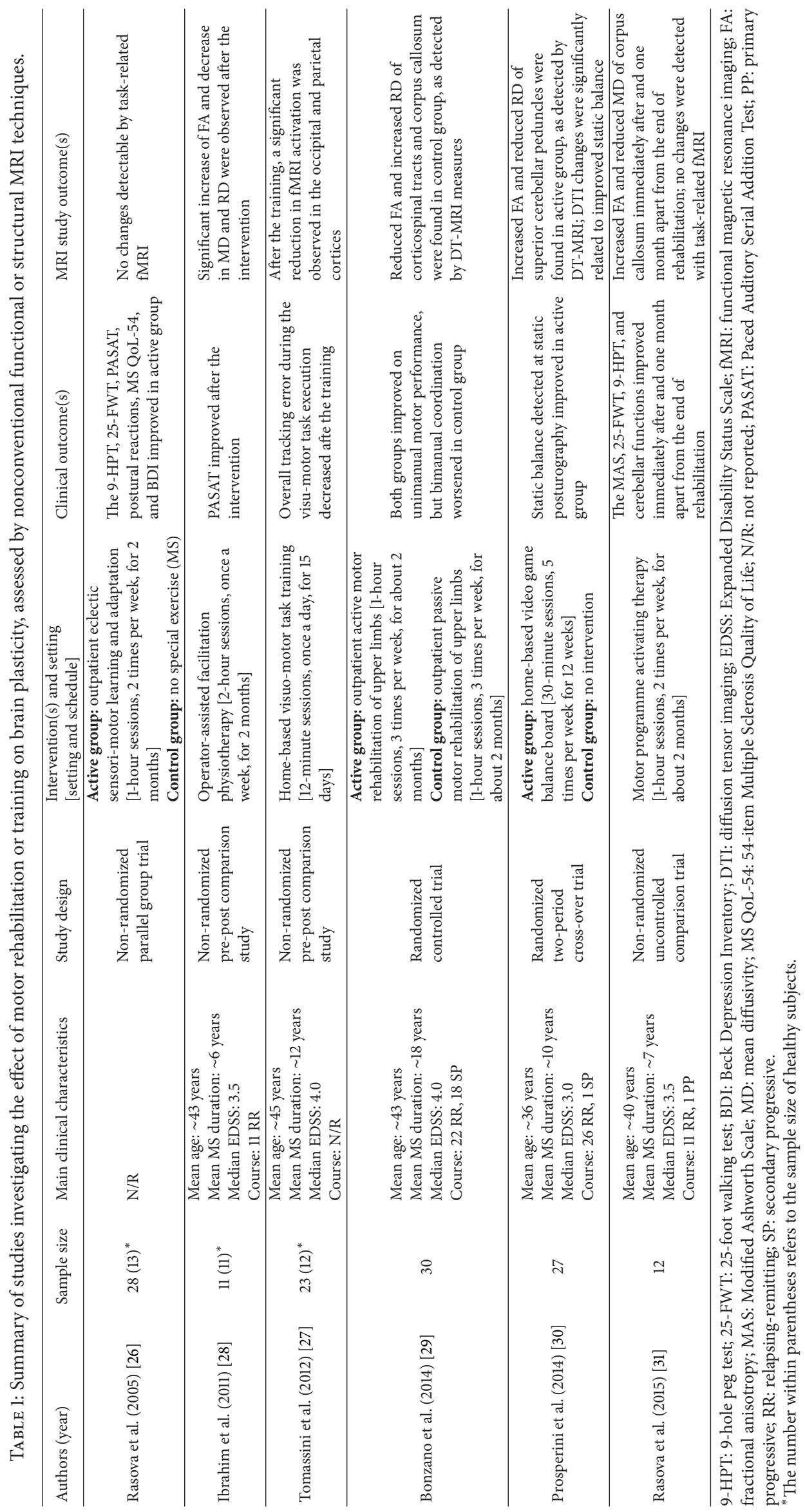




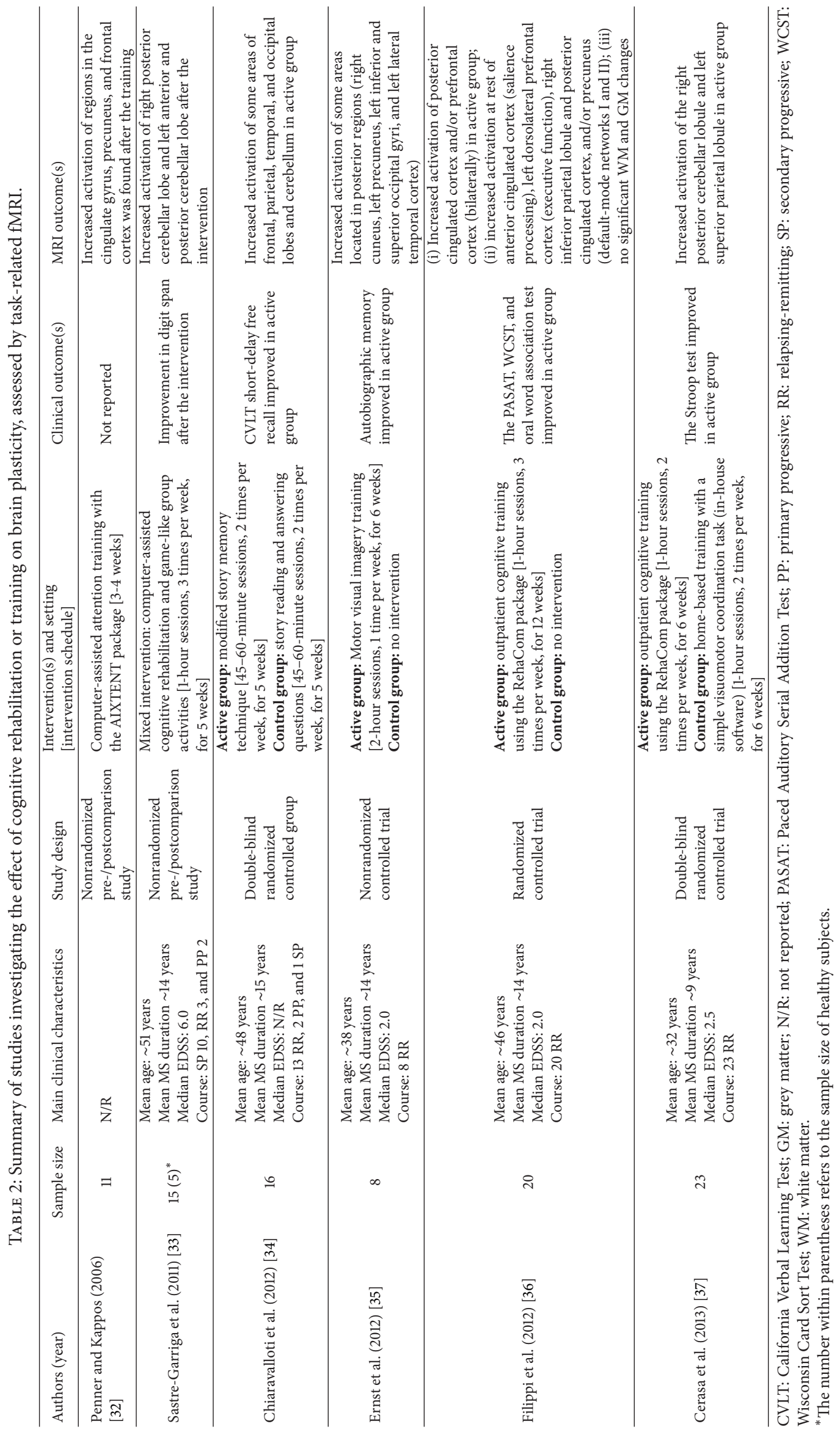




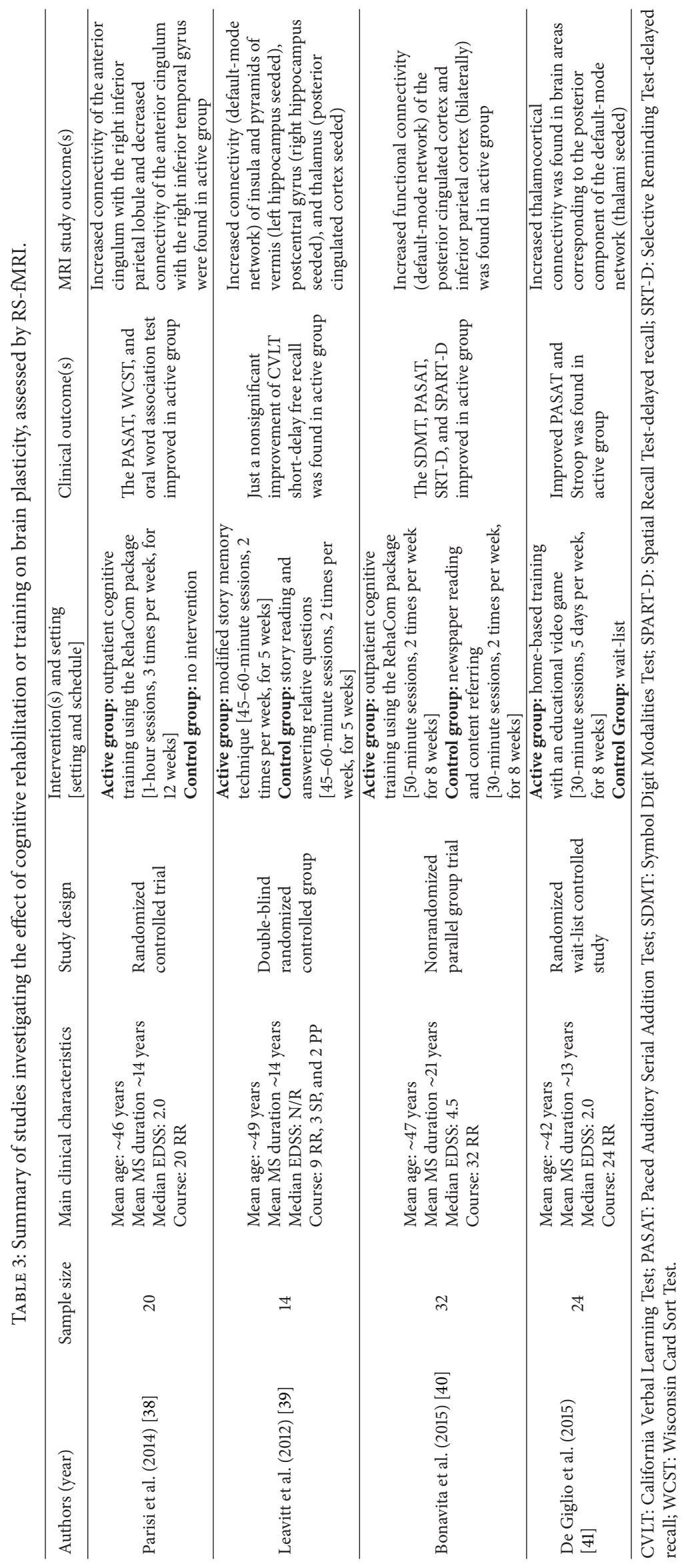


TABLE 4: Included articles rating according to the PEDro scale [25].

\begin{tabular}{|c|c|c|c|c|c|c|c|c|c|c|c|c|c|}
\hline & & $\# 1$ & $\# 2$ & $\# 3$ & $\# 4$ & $\# 5$ & $\# 6$ & $\# 7$ & $\# 8$ & $\# 9$ & $\# 10$ & $\# 11$ & Score \\
\hline \multirow{6}{*}{$\begin{array}{l}\text { Articles on motor } \\
\text { rehabilitation }\end{array}$} & Rasova et al. (2005) [26] & $\checkmark$ & $x$ & $x$ & $\checkmark$ & $x$ & $x$ & $x$ & $\checkmark$ & $\checkmark$ & $\checkmark$ & $\checkmark$ & $6 / 11$ \\
\hline & Ibrahim et al. (2011) [28] & $\checkmark$ & $x$ & $x$ & $x$ & $x$ & $x$ & $\checkmark$ & $\checkmark$ & $\checkmark$ & $\checkmark$ & $\checkmark$ & $6 / 11$ \\
\hline & Tomassini et al. (2012) [20] & $\checkmark$ & $x$ & $x$ & $x$ & $x$ & $x$ & $x$ & $\checkmark$ & $\checkmark$ & N/A & $\checkmark$ & $5 / 11$ \\
\hline & Bonzano et al. (2014) [29] & $\checkmark$ & $\checkmark$ & $\checkmark$ & $\checkmark$ & $x$ & $x$ & $x$ & $\checkmark$ & $\checkmark$ & $\checkmark$ & $\checkmark$ & $8 / 11$ \\
\hline & Prosperini et al. (2014) [30] & $\checkmark$ & $\checkmark$ & $\checkmark$ & $\checkmark$ & $x$ & $x$ & $\checkmark$ & $x$ & $\checkmark$ & $\checkmark$ & $\checkmark$ & $8 / 11$ \\
\hline & Rasova et al. (2015) [31] & $\checkmark$ & $x$ & $x$ & $x$ & $x$ & $x$ & $x$ & $\checkmark$ & $\checkmark$ & $x$ & $\checkmark$ & $4 / 11$ \\
\hline \multirow{10}{*}{$\begin{array}{l}\text { Articles on cognitive } \\
\text { rehabilitation }\end{array}$} & Penner and Kappos (2006) [32] & $\checkmark$ & $x$ & $x$ & $x$ & $x$ & $x$ & $x$ & $\checkmark$ & $\checkmark$ & N/A & $\checkmark$ & $4 / 11$ \\
\hline & Sastre-Garriga et al. (2011) [33] & $\checkmark$ & $x$ & $x$ & $x$ & $x$ & $x$ & $\checkmark$ & $\checkmark$ & $\checkmark$ & N/A & $\checkmark$ & $5 / 11$ \\
\hline & Chiaravalloti et al. (2012) [34] & $\checkmark$ & $\checkmark$ & $\checkmark$ & $\checkmark$ & $\checkmark$ & $x$ & $\checkmark$ & $\checkmark$ & $\checkmark$ & $\checkmark$ & $\checkmark$ & $10 / 11$ \\
\hline & Ernst et al. (2012) [35] & $\checkmark$ & $x$ & $x$ & $x$ & $x$ & $x$ & $x$ & $\checkmark$ & $\checkmark$ & $\checkmark$ & $\checkmark$ & $5 / 11$ \\
\hline & Filippi et al. (2012) [36] & $\checkmark$ & $\checkmark$ & $\checkmark$ & $\checkmark$ & $x$ & $x$ & $\checkmark$ & $\checkmark$ & $\checkmark$ & $\checkmark$ & $\checkmark$ & $9 / 11$ \\
\hline & Leavitt et al. (2012) [39] & $\checkmark$ & $\checkmark$ & $\checkmark$ & $\checkmark$ & $\checkmark$ & $x$ & $\checkmark$ & $\checkmark$ & $\checkmark$ & $\checkmark$ & $\checkmark$ & $10 / 11$ \\
\hline & Cerasa et al. (2013) [37] & $\checkmark$ & $\checkmark$ & $\checkmark$ & $\checkmark$ & $\checkmark$ & $x$ & $\checkmark$ & $\checkmark$ & $\checkmark$ & $\checkmark$ & $\checkmark$ & $10 / 11$ \\
\hline & Parisi et al. (2014) [38] & $\checkmark$ & $\checkmark$ & $\checkmark$ & $\checkmark$ & $x$ & $x$ & $\checkmark$ & $\checkmark$ & $\checkmark$ & $\checkmark$ & $\checkmark$ & $9 / 11$ \\
\hline & Bonavita et al. (2015) [40] & $\checkmark$ & $x$ & $x$ & $\checkmark$ & $x$ & $x$ & $\checkmark$ & $\checkmark$ & $\checkmark$ & $\checkmark$ & $\checkmark$ & $7 / 11$ \\
\hline & De Giglio et al. (2015) [41] & $\checkmark$ & $\checkmark$ & $\checkmark$ & $\checkmark$ & $x$ & $x$ & $\checkmark$ & $x$ & $\checkmark$ & $\checkmark$ & $\checkmark$ & $7 / 11$ \\
\hline
\end{tabular}

N/A: data not available.

Criterion 1: specified eligibility criteria.

Criterion 2: randomized allocation.

Criterion 3: concealed allocation.

Criterion 4: similarity between groups at baseline.

Criterion 5: blinding of subjects.

Criterion 6: blinding of therapists.

Criterion 7: blinding of assessors.

Criterion 8: outcome measures obtained from at least $85 \%$ of initially allocated subjects.

Criterion 9: all received treatment, or key outcome, was analyzed by "intention-to-treat."

Criterion 10: between-group statistical comparison.

Criterion 11: both point and variability measures provided.

weekly) [31]. Patients were clinically evaluated and scanned four times to obtain DTI data of the corpus callosum and motor task-related fMRI (flexion and extension of metacarpophalangeal joints). Follow-up data were available for 12 patients who experienced a significant improvement in some clinical scales and in DTI metrics of corpus callosum (increased FA and reduced MD) immediately after and even one month after the end of the intervention, while no relevant change was found in terms of fMRI data.

Tomassini and colleagues [27] submitted 23 patients and 13 healthy controls to short-term and long-term practice of a visuomotor task. The short-term and long-term training consisted of 12-minute training, done during the first fMRI session, and 13-minute home-based sessions, once daily, for 15 days, respectively; at the end of the training, patients and healthy subjects had a second fMRI session. From a clinical standpoint, although patients performed poorer than healthy subjects in terms of overall tracking error for the visuomotor task, the MS group improved similar to healthy group after both short-term and long-term practices, regardless of MRI measures of brain damage and disability. After the long-term practice that may be considered as equivalent to a short rehabilitative intervention, a significant reduction in taskrelated activation of the occipital and parietal cortices was found in patients. Greater long-term clinical improvement was found to be related to smaller changes in task-related activation over time in the left superior lobule and right lateral occipital cortex, but this correlation failed to reach a statistical significance. Long-term postintervention fMRI changes observed in patients differed from those found in healthy subjects who showed reduced task-related activation only in the occipital cortex. The authors concluded that adaptive plasticity is preserved even in chronically disabled patients with MS, but this plasticity is modulated by brain systems different from those acting in healthy subjects.

To investigate the possibility that rehabilitation induces microstructural changes of WM bundles involved in voluntary motor control, Bonzano and colleagues [29] randomized (in a 1:1 ratio) 30 patients with MS to receive either 2-month active, task-oriented motor rehabilitation (active group) or a 2-month passive motor rehabilitation (control group) of the upper limbs (1-hour sessions, thrice per week). Before and after rehabilitation, DTI data of the corpus callosum, left and right corticospinal tracts, and left and right superior longitudinal fasciculus were obtained. After rehabilitation, the unimanual motor performance improved in both groups, while the bimanual coordination task worsened in control group and remained stable in active group. Accordingly, reduced $\mathrm{FA}$ and increased $\mathrm{RD}$ of corticospinal tracts and corpus callosum were found in the control group, but not in 
the active one. The authors concluded that active (voluntary), but not passive, rehabilitation preserves WM integrity of brain structures specifically involved in the trained function, thus supporting the beneficial effect of task-oriented rehabilitation.

Based on data from a randomized, two-period, crossover pilot study, showing a beneficial effect of the Nintendo Wii balance board on static balance [42], Prosperini and colleagues investigated whether DTI parameters of cerebellar connections significantly changed after intervention and whether these changes correlated with clinical improvement [30]. A total of 36 patients with MS were randomized in a 1:1 ratio to two counterbalanced groups: group A received 30 -minute sessions, 5 days per week for 12 consecutive weeks of home-based video game training (intervention period), followed by a 12-week period without any specific intervention (observation period); group B was given the treatment in reverse order. Patients were clinically evaluated and MRI scanned at study beginning and at the end of the first and the second study periods; MRI data were available for 27 patients. Improved DTI measures of superior cerebellar peduncles were found after the training (increased FA and reduced $\mathrm{RD}$ ), suggesting the occurrence of activity-dependent myelomodulation in partially damaged pathways (see Figure 2). These microstructural changes were also significantly related to clinical improvement of static balance, supporting the hypothesis that structural plasticity may be enhanced in brain areas specifically involved in the function trained with highintensity, task-oriented rehabilitation. However, there was no retention of training-induced improvement in clinical and MRI measures.

3.2. Brain Plasticity Enhanced by Cognitive Rehabilitation. In a preliminary study, 11 patients with MS suffering from mild to severe cognitive impairment were submitted to a 3-4-week intervention with the AIXTENT software to train alertness, divided attention, selective attention, and vigilance [32]. By comparing pre- and posttraining clinical findings and taskrelated fMRI features, Penner and Kappos demonstrated both a clinical improvement and an increased activation of regions in the cingulate gyrus, precuneus, and frontal cortex; all these areas are known to be involved in a network functionally related to attention processing.

The effect of a mixed intervention (game-like group activities and computer-aided training) was investigated by Sastre-Garriga and colleagues [33] in an open-label, proofof-concept trial. Fifteen patients with MS underwent an extensive neuropsychological evaluation and were scanned to obtain task-related (PASAT) fMRI before and after a 5week run-in period. Further clinical and fMRI data were collected at the end of a 5-week cognitive training period (1hour sessions, thrice per week). Five healthy subjects who were scanned at the same time-points served as healthy controls. After the training, patients exhibited an improved performance in backward version of digit span and increased fMRI activation in right posterior lobe (uvula and declive) and left anterior and posterior lobes of cerebellum (declive and culmen). However, clinical findings and fMRI changes did not significantly correlate, likely because the study was

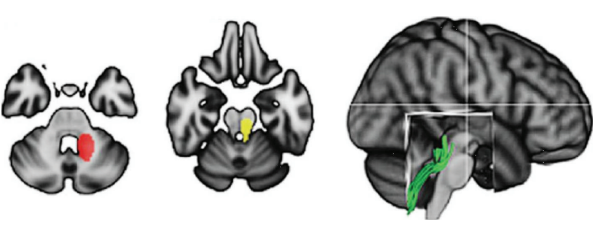

FIGURE 2: Regions of interest outlining the superior cerebellar peduncle dissected by means of streamline tractography; this white matter bundle showed significant changes indicating improved structural integrity following the 12-week home-based training using the Nintendo Wii balance board (modified from [30]).

underpowered to detect relevant clinical changes owing the small sample size [33].

A double-blind, placebo-controlled, randomized clinical trial was designed to investigate changes in brain activation following modified Story Memory Technique (mSMT), a rehabilitative approach used for treating new learning and memory deficits [34]. A total of 16 patients were randomized either to a 5-week treatment using mSMT (active group) or to story reading and answering related questions (control group), with the same schedule (45-60-minute sessions, twice per week). Both groups were scanned to obtain taskrelated fMRI data during list-learning and word-recognition tasks. The proportion of patients who improved memory performance on California Verbal Learning Test short-delay free recall after the intervention was greater in the active than in the control group. Compared to controls, patients in the active group showed an increased activation in some areas of frontal, parietal, temporal, and occipital cortices and in the cerebellum. There was also a significant correlation between improved memory performance and increased activation of the right middle frontal gyrus, which is known to be associated with visual and context-dependent learning.

Some patients originally enrolled in this latter study underwent also RS-fMRI, in order to explore FC using the left and the right hippocampus (implicated in memory function) and posterior cingulated cortex (involved in the default-mode network) as seeding points [39]. Out of the two analyses performed, the less conservative one showed that after the training the active group had an increased connectivity between the left hippocampus, the insula, and pyramids of vermis, between the right hippocampus and the postcentral gyrus, and between the posterior cingulated cortex and thalamus.

Brain activation changes following a 6-week visual imagery training (2-hour sessions, once weekly) were investigated in 4 patients with MS [35]. They underwent preand posttraining clinical evaluations and task-related fMRI (evocation of specific personal memories). A significant improvement of autobiographic memory performance, coupled with increased activation of posterior cerebral areas specifically involved in memory retrieval (right cuneus, left precuneus, left inferior and superior occipital gyri, and left lateral temporal cortex), was found after the rehabilitation.

Cerasa and colleagues [37] performed a randomized, double-blind, controlled trial in which 26 patients with MS were allocated to receive a 6 -week computer-aided training 
(1-hour sessions, twice per week) either with the RehaCom package, a modular system developed to treat a wide spectrum of cognitive functioning, including alertness, attention, memory, executive functions, and visual field (active group), or with a simple visuomotor coordination task (control group). Before and after the intervention, both groups were clinically examined and scanned to obtain task-related fMRI data (visual PASAT). Performance at Stroop test improved in the active group only, which also showed increased activation of brain areas subserving refreshing phonological stimuli and short-term information storage, that is, the right posterior cerebellar lobule and left superior parietal lobule.

Filippi and colleagues [36] used functional and structural MRI to investigate brain changes after a 12-week computer-assisted training with RehaCom. Twenty patients with MS were randomly allocated either to the active group $(n=10)$ or to the control group, which did not undergo any intervention $(n=10)$. Before and after the 12-week study period, both groups were assessed by a complete neuropsychological evaluation and scanned to map changes in WM and GM structures and to obtain task-related fMRI (Stroop test) and RS-fMRI data. The active group showed a clinical improvement in some tests of attention, information processing, and executive functions, an increased activation of posterior cingulated cortex and/or precuneus and dorsolateral prefrontal cortex (bilaterally) during the task-related fMRI, and increased RS-FC of the anterior cingulated cortex (salience processing), left dorsolateral prefrontal cortex (executive function), right inferior parietal lobule, posterior cingulated cortex, and/or precuneus (default-mode network). Neither WM nor GM microarchitecture, assessed with DTI and voxel-based morphometry [43], was impacted by the rehabilitation. The authors concluded that rehabilitation of attention, information processing speed, and executive function enhance recruitment of brain networks subserving the trained functions.

In another study, data from the same population were reanalyzed using the anterior cingulated cortex as seed to explore its RS-FC [38]. At follow-up, the anterior cingulum showed an increased FC with the right inferior parietal lobule and decreased FC with the right inferior temporal gyrus in the active group only; some of these FC changes were significantly related to improved PASAT scores after the training.

Bonavita and colleagues [40] performed a nonrandomized parallel-group trial in which 18 patients were trained using the RehaCom package (active group) and 14 patients were submitted to newspaper reading and content referring for 8 consecutive weeks. Both groups underwent an extensive neuropsychological evaluation and RS-fMRI study at entry and at the end of follow-up. Several neuropsychological tests of information processing speed and verbal and visual sustained memory improved in the active, but not in the control, group after the 8-week study period. Likewise, increased RSFC in the posterior cingulated cortex and inferior parietal cortex bilaterally (subserving the default-mode network) was found in the active group.

Lastly, De Giglio and colleagues performed a randomized, wait-list controlled study to investigate the effectiveness of

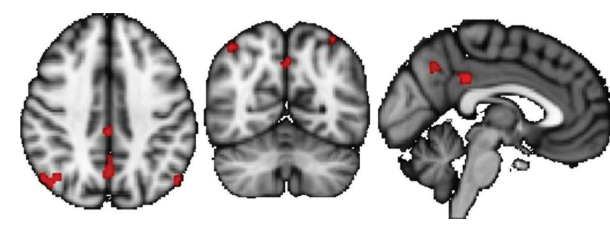

Figure 3: Areas of increased thalamic connectivity (posterior cingulate gyrus, precuneus, and lateral parietal cortex, bilaterally) following the 8-week home-based training using the Nintendo Dr. Kawashima Brain Training (modified from [41], courtesy of Dr. De Giglio).

8-week home-based playing period with the Dr. Kawashima Nintendo Brain Training, an educational video game aimed at training memory, attention, visuospatial processing, and calculations. The active group exhibited a significant improvement in sustained/divided attention and some aspects of executive functions [44]. In a post hoc analysis recently submitted for publication, 24 patients enrolled in the original trial underwent RS-fMRI before and after the cognitive training [41]. We found that this type of cognitive rehabilitative training induced an increased thalamic FC in brain areas corresponding to the posterior component of the defaultmode network (cingulum, precuneus, and bilateral parietal cortex) and a decreased connectivity in the vermis and left dorsolateral prefrontal cortex (see Figure 3). Moreover, positive correlations were found between improved cognitive performance (PASAT, Symbol Digit Modalities Test, and Stroop test) and increased FC in areas belonging to the default-mode network.

\section{Discussion}

Brain plasticity represents the substrate for interventions promoting functional recovery, by means of neural restoration or compensation [12, 20,21]. Findings from the present systematic review suggest that there is MRI-based evidence that functional or structural plasticity occurred following motor or cognitive rehabilitation in patients with MS. In addition, some studies also showed relevant relationship between improved function and MRI-detected brain changes $[27,30,34,36-38,40,41]$. This latter feature supports the notion that training-induced plasticity is specifically linked to the trained function and it is not merely a general effect of any rehabilitation.

Although they differed from each other, studies on motor rehabilitation support the notion that brain plasticity is enhanced by task-dependent and target-selected training [27, $29,30]$, rather than by an "holistic" approach [26]. Improved microstructural properties of corpus callosum were found following high-intensity, repetitive training of motor functions involving the lower limbs and task-oriented exercises aimed at improving upper limb functions [28, 29, 31]. Callosal fibers connect homologous cortical areas of the two hemispheres, thus subserving a wide range of motor and cognitive function, including gait and bimanual coordination [45-48]. Favourable changes in the microarchitecture of the superior 
cerebellar connections were reported after a video gamebased training of balance [30]. Superior cerebellar peduncles mainly contain output fibers projecting from cerebellum to the neocortex, contributing to a high-level sensory weighting for postural control [49]. However, the interpretation of DTI parameters in relation to pathological changes derived is still controversial [50], and some authors argued that RD does not selectively measures demyelination due to MS but represents more complex tissue changes [51]. In addition, the reliability and sensitivity to changes of DTI measures are not well elucidated yet [19].

Studies on cognitive rehabilitation are somewhat more consistent than those on motor rehabilitation, not only in terms of trained functions but also in their results. The majority of intervention strategies consisted of computerassisted training of attention, short-term memory, and executive functions [32, 33, 36-38, 40, 41]. Despite some differences regarding the neuropsychological scales and clinical outcome measures adopted, task-related fMRI and RS-fMRI findings are quite consistent, pointing out the role of some specific brain regions such as the cingulated cortex $[32,34,36,38$, $40,41]$, precuneus $[32,34-36,41]$, and cerebellum [33, 34, $37,39]$. The cingulated cortex is known to cover emotion formation and processing, learning, and memory, thus linking behavioral outcomes to motivational learning [52-54]. The precuneus is involved in episodic memory and visuospatial imagery and it has been suggested to be a specific target for visual mirror therapy and virtual reality-based rehabilitation $[55,56]$. Being connected with many association networks [57], the cerebellum has been now recognized to be not only involved in motor planning and learning, but also in different cognitive domains, including attention, memory, and learning, executive control, language, and visuospatial function $[58,59]$.

Despite some encouraging findings reported above, the studies included in the present systematic review suffer from several drawbacks, mainly concerning the small sample size and the absence of a nonactive control group, blindness, and/or randomization in the study design. Moreover, the selected articles are not comparable because clinical outcome measures, MRI biomarkers, and intervention are not standardized.

Only few articles report data about the occurrence of acute relapses and disability progression [28, 30, 37, 38, 41] or information on disease-modifying and symptomatic treatments taken by patients while on study [28, 29]. However, this should have not biased findings/interpretation of the included studies for several reasons: (i) no patients relapsed or experienced disability progression (when reported) [28, $30,37,38,41]$; (ii) it is very unlikely that relapses and disability progression might have occurred, given the short duration of the studies (from a minimum of 15 days to a maximum of 24 weeks) in the remaining studies [26, 27, 29, 31-36, 39, 40]; (iii) as per inclusion criteria only patients in a stable phase of the disease were enrolled; (iv) the randomization procedure (when applied) should have prevented any imbalance in known and unknown baseline characteristics between treatment and control groups $[29,30$, $34,36-39,41]$.
The lack of statistical inferences aimed at exploring correlations between imaging results and clinical outcomes represents another major limit of some studies [26, 28, 29, $31,32,34,35,40]$ since there is recommendation that MRI changes following the rehabilitative interventions should be quantified and compared with clinically relevant, sensitive, and reproducible outcomes [20,60].

Postintervention study phases were planned only rarely, but they may provide important information about the retention of rehabilitation-induced clinical and MRI improvements, especially for defining the most appropriate duration and timing of rehabilitation. Therefore, efforts for future research should be focused on establishing (i) the most appropriate strategies for effective rehabilitation; (ii) standardised, valid, and reliable endpoints to assess the efficacy of rehabilitation, taking into account the concept of ecological validity and patient-centered outcomes; and (iii) clinical and MRI measures that most effectively detect the occurrence of beneficial brain plasticity after specific training.

Another new intriguing field of research that is worth developing encompasses the possibility of combining rehabilitation with pharmacologic treatments or neuromodulation, to obtain a synergistic or even a more than additive effect on brain plasticity, as demonstrated in other pathological conditions [61-63].

In conclusion, the current knowledge about the rehabilitation-induced brain plasticity in MS is still fragmented and incomplete. The ultimate goal should be to demonstrate, at an evidence-based level, that effective rehabilitation favourably affects the brain structures, improves the trained function, and promotes the patient's quality of life.

\section{Conflict of Interests}

The authors declare that there is no conflict of interests regarding the publication of this paper.

\section{References}

[1] A. Compston and A. Coles, "Multiple sclerosis," The Lancet, vol. 359, no. 9313, pp. 1221-1231, 2002.

[2] R. Dutta and B. D. Trapp, "Mechanisms of neuronal dysfunction and degeneration in multiple sclerosis," Progress in Neurobiology, vol. 93, no. 1, pp. 1-12, 2011.

[3] F. D. Lublin, M. Baier, and G. Cutter, "Effect of relapses on development of residual deficit in multiple sclerosis," Neurology, vol. 61, no. 11, pp. 1528-1532, 2003.

[4] A. Traboulsee, "MRI relapses have significant pathologic and clinical implications in multiple sclerosis," Journal of the Neurological Sciences, vol. 256, no. 1, pp. S19-S22, 2007.

[5] M. S. Freedman, "Time is brain' also in multiple sclerosis," Multiple Sclerosis, vol. 15, no. 10, pp. 1133-1134, 2009.

[6] C. H. Polman, S. C. Reingold, B. Banwell et al., "Diagnostic criteria for multiple sclerosis: 2010 revisions to the McDonald criteria," Annals of Neurology, vol. 69, no. 2, pp. 292-302, 2011.

[7] P. van Asch, "Impact of mobility impairment in multiple sclerosis 2-patients' perspectives," European Neurological Review, vol. 6, no. 2, pp. 115-120, 2011. 
[8] R. H. B. Benedict and R. Zivadinov, "Risk factors for and management of cognitive dysfunction in multiple sclerosis," Nature Reviews Neurology, vol. 7, no. 6, pp. 332-342, 2011.

[9] C. Leone, F. Patti, and P. Feys, "Measuring the cost of cognitivemotor dual tasking during walking in multiple sclerosis," Multiple Sclerosis Journal, vol. 21, no. 2, pp. 123-131, 2015.

[10] J. Kesselring and S. Beer, "Symptomatic therapy and neurorehabilitation in multiple sclerosis," The Lancet Neurology, vol. 4, no. 10, pp. 643-652, 2005.

[11] S. Beer, F. Khan, and J. Kesselring, "Rehabilitation interventions in multiple sclerosis: an overview," Journal of Neurology, vol. 259, no. 9, pp. 1994-2008, 2012.

[12] J. A. Kleim, "Neural plasticity and neurorehabilitation: teaching the new brain old tricks," Journal of Communication Disorders, vol. 44, no. 5, pp. 521-528, 2011.

[13] A. Pascual-Leone, A. Amedi, F. Fregni, and L. B. Merabet, "The plastic human brain cortex," Annual Review of Neuroscience, vol. 28, no. 1, pp. 377-401, 2005.

[14] D. Zeller and J. Classen, "Plasticity of the motor system in multiple sclerosis," Neuroscience, vol. 283, pp. 222-230, 2014.

[15] P. S. Giacomini and D. L. Arnold, "Non-conventional MRI techniques for measuring neuroprotection, repair and plasticity in multiple sclerosis," Current Opinion in Neurology, vol. 21, no. 3, pp. 272-277, 2008.

[16] P. Pantano, C. Mainero, and F. Caramia, "Functional brain reorganization in multiple sclerosis: evidence from fMRI studies," Journal of Neuroimaging, vol. 16, no. 2, pp. 104-114, 2006.

[17] R. Sacco, S. Bonavita, F. Esposito, G. Tedeschi, and A. Gallo, "The contribution of resting state networks to the study of cortical reorganization in MS," Multiple Sclerosis International, vol. 2013, Article ID 857807, 7 pages, 2013.

[18] S. Mori and J. Zhang, "Principles of diffusion tensor imaging and its applications to basic neuroscience research," Neuron, vol. 51, no. 5, pp. 527-539, 2006.

[19] E. Sbardella, F. Tona, N. Petsas, and P. Pantano, "DTI measurements in multiple sclerosis: evaluation of brain damage and clinical implications," Multiple Sclerosis International, vol. 2013, Article ID 671730, 11 pages, 2013.

[20] V. Tomassini, P. M. Matthews, A. J. Thompson et al., "Neuroplasticity and functional recovery in multiple sclerosis," Nature Reviews Neurology, vol. 8, no. 11, pp. 635-646, 2012.

[21] C. Mainero, P. Pantano, F. Caramia, and C. Pozzilli, "Brain reorganization during attention and memory tasks in multiple sclerosis: insights from functional MRI studies," Journal of the Neurological Sciences, vol. 245, no. 1-2, pp. 93-98, 2006.

[22] M. Filippi, A. Charil, M. Rovaris, M. Absinta, and M. A. Rocca, "Insights from magnetic resonance imaging," in Handbook of Clinical Neurology, vol. 122 of Multiple Sclerosis and Related Disorders, chapter 6, pp. 115-149, 2014.

[23] J. Deluca and U. Nocentini, "Neuropsychological, medical and rehabilitative management of persons with multiple sclerosis," NeuroRehabilitation, vol. 29, no. 3, pp. 197-219, 2011.

[24] D. Moher, A. Liberati, J. Tetzlaff, D. G. Altman, and PRISMA Group, "Preferred reporting items for systematic reviews and meta-analyses: the PRISMA statement," British Medical Journal, vol. 339, Article ID b2535, 2009.

[25] C. G. Maher, C. Sherrington, R. D. Herbert, A. M. Moseley, and M. Elkins, "Reliability of the PEDro scale for rating quality of randomized controlled trials," Physical Therapy, vol. 83, no. 8, pp. 713-721, 2003.
[26] K. Rasova, J. Krasenksy, E. Havrdova et al., "Is it possible to actively and purposely make use of plasticity and adaptability in the neurorehabilitation treatment of multiple scelorsis patients? A pilot project," Clinical Rehabilitation, vol. 19, no. 2, pp. 170-181, 2005.

[27] V. Tomassini, H. Johansen-Berg, S. Jbabdi et al., "Relating brain damage to brain plasticity in patients with multiple sclerosis," Neurorehabilitation \& Neural Repair, vol. 26, no. 6, pp. 581-593, 2012.

[28] I. Ibrahim, J. Tintera, A. Skoch et al., "Fractional anisotropy and mean diffusivity in the corpus callosum of patients with multiple sclerosis: the effect of physiotherapy," Neuroradiology, vol. 53, no. 11, pp. 917-926, 2011.

[29] L. Bonzano, A. Tacchino, G. Brichetto et al., "Upper limb motor rehabilitation impacts white matter microstructure in multiple sclerosis," NeuroImage, vol. 90, pp. 107-116, 2014.

[30] L. Prosperini, F. Fanelli, N. Petsas et al., "Multiple sclerosis: changes in microarchitecture of white matter tracts after training with a video game balance board," Radiology, vol. 273, no. 2, pp. 529-538, 2014.

[31] K. Rasova, M. Prochazkova, J. Tintera, I. Ibrahim, D. Zimova, and I. Stetkarova, "Motor programme activating therapy influences adaptive brain functions in multiple sclerosis: clinical and MRI study," International Journal of Rehabilitation Research, vol. 38, no. 1, pp. 49-54, 2015.

[32] I.-K. Penner and L. Kappos, "Retraining attention in MS," Journal of the Neurological Sciences, vol. 245, no. 1-2, pp. 147-151, 2006.

[33] J. Sastre-Garriga, J. Alonso, M. Renom et al., "A functional magnetic resonance proof of concept pilot trial of cognitive rehabilitation in multiple sclerosis," Multiple Sclerosis, vol. 17, no. 4, pp. 457-467, 2011.

[34] N. D. Chiaravalloti, G. Wylie, V. Leavitt, and J. DeLuca, "Increased cerebral activation after behavioral treatment for memory deficits in MS," Journal of Neurology, vol. 259, no. 7, pp. 1337-1346, 2012.

[35] A. Ernst, A. Botzung, D. Gounot et al., "Induced brain plasticity after a facilitation programme for autobiographical memory in multiple sclerosis: a preliminary study," Multiple Sclerosis International, vol. 2012, Article ID 820240, 12 pages, 2012.

[36] M. Filippi, G. Riccitelli, F. Mattioli et al., "Multiple sclerosis: effects of cognitive rehabilitation on structural and functional MR imaging measures-an explorative study," Radiology, vol. 262, no. 3, pp. 932-940, 2012.

[37] A. Cerasa, M. C. Gioia, P. Valentino et al., "Computer-assisted cognitive rehabilitation of attention deficits for multiple sclerosis: a randomized trial with fMRI correlates," Neurorehabilitation and Neural Repair, vol. 27, no. 4, pp. 284-295, 2013.

[38] L. Parisi, M. A. Rocca, P. Valsasina, L. Panicari, F. Mattioli, and M. Filippi, "Cognitive rehabilitation correlates with the functional connectivity of the anterior cingulate cortex in patients with multiple sclerosis," Brain Imaging and Behavior, vol. 8, no. 3, pp. 387-393, 2014.

[39] V. M. Leavitt, G. R. Wylie, P. A. Girgis, J. Deluca, and N. D. Chiaravalloti, "Increased functional connectivity within memory networks following memory rehabilitation in multiple sclerosis," Brain Imaging and Behavior, vol. 8, no. 3, pp. 394-402, 2012.

[40] S. Bonavita, R. Sacco, M. D. Corte et al., "Computer-aided cognitive rehabilitation improves cognitive performances and induces brain functional connectivity changes in relapsing 
remitting multiple sclerosis patients: an exploratory study," Journal of Neurology, vol. 262, no. 1, pp. 91-100, 2015.

[41] L. de Giglio, F. Tona, N. Petsas et al., "Changes in thalamic resting-state functional connectivity induced by a home-based cognitive rehabilitation program in patients with multiple sclerosis," in Proceedings of the 20th Annual RIMS Conference, Milan, Italy, April 2015.

[42] L. Prosperini, D. Fortuna, C. Giannì, L. Leonardi, M. R. Marchetti, and C. Pozzilli, "Home-based balance training using the Wii balance board: a randomized, crossover pilot study in multiple sclerosis," Neurorehabilitation and Neural Repair, vol. 27, no. 6, pp. 516-525, 2013.

[43] J. Ashburner and K. J. Friston, "Voxel-based morphometry-the methods," NeuroImage, vol. 11, no. 6 I, pp. 805-821, 2000.

[44] L. de Giglio, F. de Luca, L. Prosperini et al., "A low-cost cognitive rehabilitation with a commercial video game improves sustained attention and executive functions in multiple sclerosis: a pilot study," Neurorehabilitation \& Neural Repair, 2014.

[45] S. W. Kennerley, J. Diedrichsen, E. Hazeltine, A. Semjen, and R. B. Ivry, "Callosotomy patients exhibit temporal uncoupling during continuous bimanual movements," Nature Neuroscience, vol. 5, no. 4, pp. 376-381, 2002.

[46] B. W. Fling, J. A. Bernard, J. Bo, and J. Langan, "Corpus callosum and bimanual coordination in multiple sclerosis," Journal of Neuroscience, vol. 28, no. 29, pp. 7248-7249, 2008.

[47] A. Ozturk, S. A. Smith, E. M. Gordon-Lipkin et al., "MRI of the corpus callosum in multiple sclerosis: association with disability," Multiple Sclerosis, vol. 16, no. 2, pp. 166-177, 2010.

[48] C. Ryberg, E. Rostrup, O. B. Paulson et al., "Corpus callosum atrophy as a predictor of age-related cognitive and motor impairment: a 3-year follow-up of the LADIS study cohort," Journal of the Neurological Sciences, vol. 307, no. 1-2, pp. 100105, 2011.

[49] M. Manto, J. M. Bower, A. B. Conforto et al., "Consensus paper: roles of the cerebellum in motor control-the diversity of ideas on cerebellar involvement in movement," Cerebellum, vol. 11, no. 2, pp. 457-487, 2012.

[50] C. Beaulieu, "The basis of anisotropic water diffusion in the nervous system - a technical review," NMR in Biomedicine, vol. 15, no. 7-8, pp. 435-455, 2002.

[51] S. Cader, H. Johansen-Berg, M. Wylezinska et al., "Discordant white matter $\mathrm{N}$-acetylasparate and diffusion MRI measures suggest that chronic metabolic dysfunction contributes to axonal pathology in multiple sclerosis," NeuroImage, vol. 36, no. 1, pp. 19-27, 2007.

[52] B. Faw, "Pre-frontal executive committee for perception, working memory, attention, long-term memory, motor control, and thinking: a tutorial review," Consciousness and Cognition, vol. 12, no. 1, pp. 83-139, 2003.

[53] P. J. Morgane, J. R. Galler, and D. J. Mokler, "A review of systems and networks of the limbic forebrain/limbic midbrain," Progress in Neurobiology, vol. 75, no. 2, pp. 143-160, 2005.

[54] B. Y. Hayden and M. L. Platt, "Neurons in anterior cingulate cortex multiplex information about reward and action," The Journal of Neuroscience, vol. 30, no. 9, pp. 3339-3346, 2010.

[55] A. E. Cavanna and M. R. Trimble, "The precuneus: a review of its functional anatomy and behavioural correlates," Brain, vol. 129, no. 3, pp. 564-583, 2006.

[56] C. Dohle, K. M. Stephan, J. T. Valvoda et al., "Representation of virtual arm movements in precuneus," Experimental Brain Research, vol. 208, no. 4, pp. 543-555, 2011.
[57] R. L. Buckner, "The cerebellum and cognitive function: 25 years of insight from anatomy and neuroimaging," Neuron, vol. 80, no. 3, pp. 807-815, 2013.

[58] D. Timmann and I. Daum, "Cerebellar contributions to cognitive functions: a progress report after two decades of research," Cerebellum, vol. 6, no. 3, pp. 159-162, 2007.

[59] L. F. Koziol, D. Budding, N. Andreasen et al., "Consensus paper: the cerebellum's role in movement and cognition," Cerebellum, vol. 13, no. 1, pp. 151-177, 2014.

[60] I. Lipp and V. Tomassini, "Neuroplasticity and motor rehabilitation in multiple sclerosis," Frontiers in Neurology, 2015.

[61] H. Duffau, "Brain plasticity: from pathophysiological mechanisms to therapeutic applications," Journal of Clinical Neuroscience, vol. 13, no. 9, pp. 885-897, 2006.

[62] S.-L. Liew, E. Santarnecchi, E. R. Buch, and L. G. Cohen, "Non-invasive brain stimulation in neurorehabilitation: local and distant effects for motor recovery," Frontiers in Human Neuroscience, vol. 8, article 378, 2014.

[63] D. M. Martin, R. Liu, A. Alonzo, M. Green, and C. K. Loo, "Use of transcranial direct current stimulation (tDCS) to enhance cognitive training: effect of timing of stimulation," Experimental Brain Research, vol. 232, no. 10, pp. 3345-3351, 2014. 

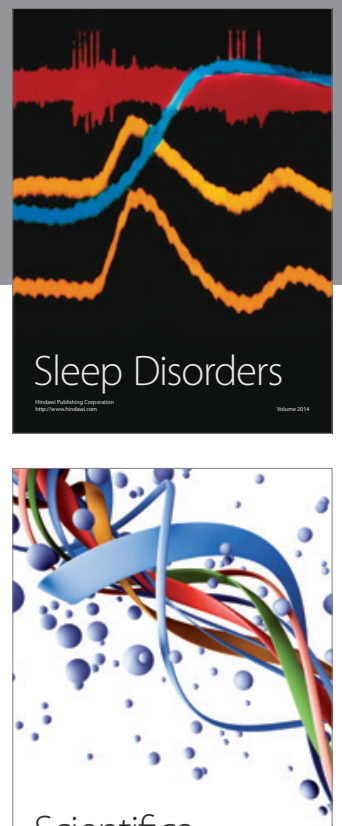

Scientifica
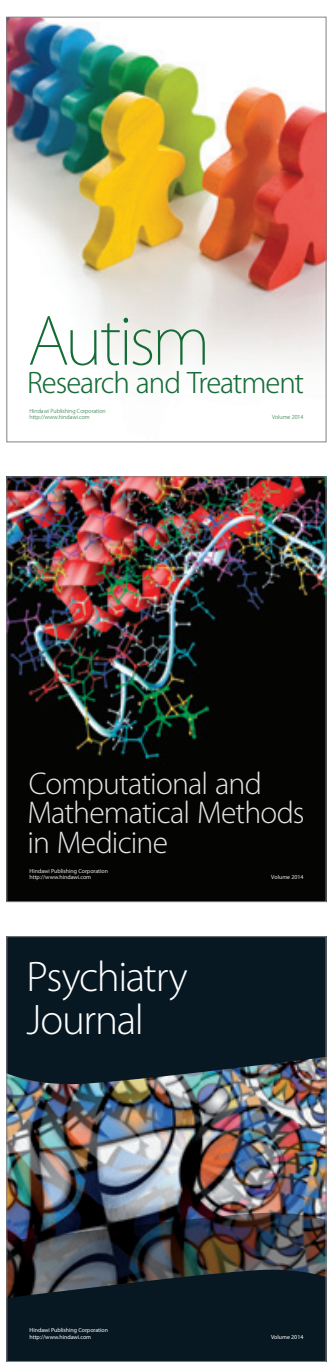
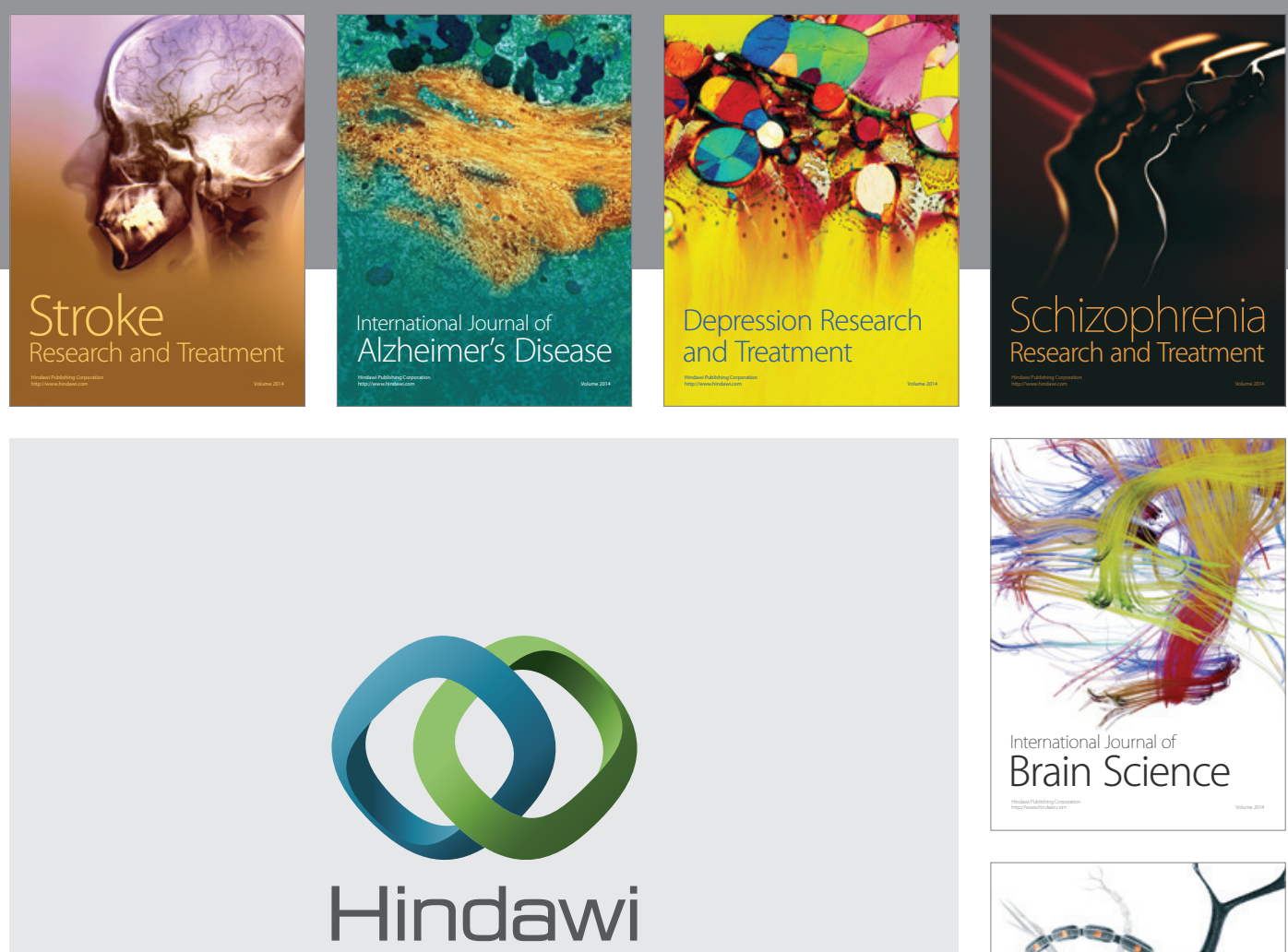

Submit your manuscripts at

http://www.hindawi.com
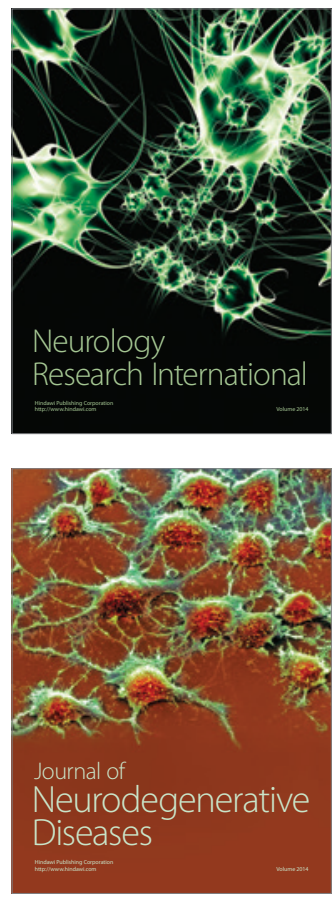

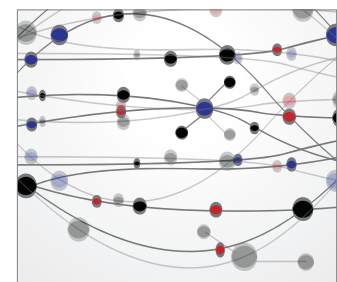

The Scientific World Journal
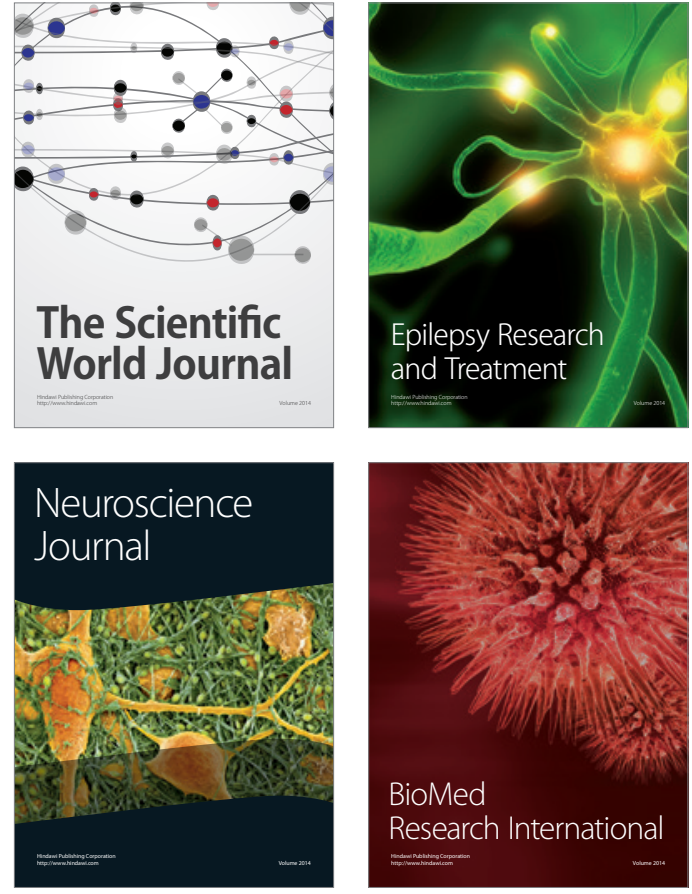

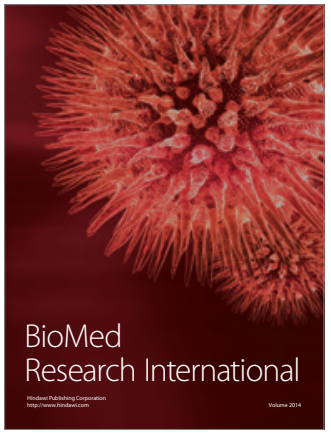

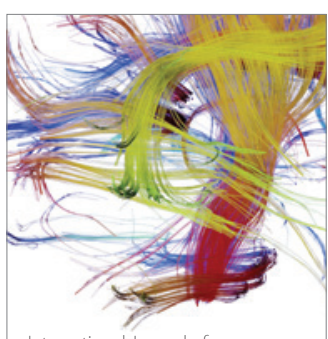

Brain Science

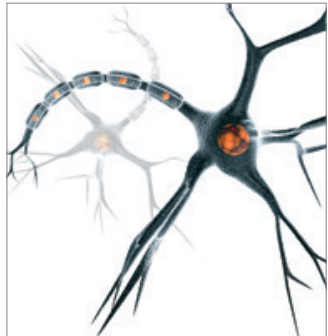

Neural Plasticity
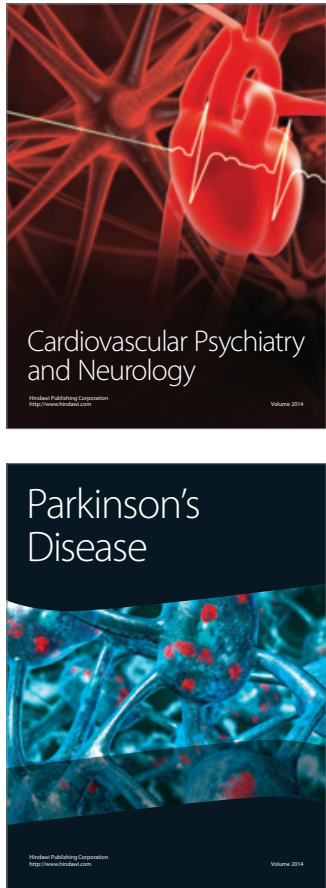